\title{
Analysis of A Neural Oscillator Model with Plasticity for Treatment of Tinnitus
}

\author{
K. Fujimoto ${ }^{1}$, H. Nagashino ${ }^{1}$, Y. Kinouchi ${ }^{2}$, A. A. Danesh ${ }^{3}$, and A. S. Pandya ${ }^{4}$ \\ ${ }^{1}$ Faculty of Medicine, The University of Tokushima, Tokushima, Japan \\ ${ }^{2}$ Institute of Technology and Science, The University of Tokushima, Tokushima, Japan \\ ${ }^{3}$ College of Education, Florida Atlantic University, Florida, USA \\ ${ }^{4}$ College of Engineering and Computer Science, Florida Atlantic University, Florida, USA
}

\begin{abstract}
Tinnitus is a symptom of perceiving phantom sounds. The majority of tinnitus cases are caused by misinterpreting null sounds from ears as significant nervous signals in the cerebral limbic system. There are two typical sound therapies for tinnitus: Tinnitus Masking (TM) and Tinnitus Retraining Therapy (TRT). Their effects have been discussed from clinical assessments. To account for the mechanism of perceiving tinnitus and the clinical effects from the viewpoint of engineering, this paper describes a neural oscillator model with a plastic coupling for a cerebral limbic system and its dynamic behavior. We observed a bi-stable state such that a stable equilibrium and a stable oscillation coexist in a certain range of parameters. It was also discovered that the value of the plastic coupling changes by external stimulation, and then the oscillation is inhibited. This could explain the fact that the retrained cerebral limbic system temporarily stops perceiving tinnitus after sound therapy.
\end{abstract}

Keywords - tinnitus, sound therapy, neural oscillator model, plastic coupling, inhibition of oscillation

\section{INTRODUCTION}

Tinnitus affects about $17 \%$ of the general population. The $15 \%$ of sufferers from tinnitus reported frequent or continuous tinnitus in a survey for a large number of people [1], and the most sufferers also have the other ear disease. Thus it is hypothesized that malfunction of cochlea is one of its causes, and then tinnitus appears in their brain [2]. A mechanism of tinnitus generation was proposed from the viewpoint of neurophysiology [3]. According to the neurophysiological model, tinnitus is caused by a vicious cycle of nervous signal among subcortex, auditory cortex, cerebral limbic system, and autonomic system. Because human emotion is managed by the cerebral limbic system, it is assumed that infliction for tinnitus disappears by retraining the system with plasticity.

There are two typical sound therapies: Tinnitus Masking (TM) [4] and Tinnitus Retraining Therapy (TRT) [2, 3]. In the former therapy, sound of tinnitus is masked by external sound stimulation. In the latter therapy, the cerebral limbic system is retrained by external sound stimulation so that tinnitus disappears. Its retraining is subject to plastic modification of synapses. Note that TRT consists of the sound therapy and psychotherapy by directive counseling; it has been obtained high assessment for its clinical effect [5].

The purpose of our study is to explain the mechanism of tinnitus generation and the clinical effects of the two sound therapies from the viewpoint of engineering. In this paper we propose a neural oscillator model with a plastic coupling for the cerebral limbic system. It is also discussed that oscillation and its inhibition by external stimulation in the model.

\section{TINNITUS}

\section{A. Types of tinnitus}

Tinnitus is classified into tinnitus with physical oscillation in ear and without such oscillation. The former tinnitus occurs outside of cochlear. Its causal factor is malfunction of the middle ear, e.g., the chained ear ossicles are out of alignment. Thus, surgical treatment can cure the tinnitus.

On the other hand, the latter tinnitus is caused by perceiving insignificant nervous signal generated in the inner ears and/or auditory nervous system as significant signal. Then, it is hypothesized that a vicious cycle of nervous signal in the brain is generated by hyperacusis for the insignificant signal. A neurophysiological mechanism for generation of the tinnitus has been proposed [4], and then this paper focuses on only this type of tinnitus.

\section{B. Neurophysiological model of tinnitus generation}

We describe the mechanism of tinnitus generation. Its neurophysiological model is shown in Fig. 1. Abnormal nervous signal (insignificant signal) generated in the peripheral auditory nervous system are perceived by the subcortex. The cerebral cortex (auditory cortex) recognizes the perceived signal, and then the signal is evaluated as negative. Thus, the cerebral limbic system produces unpleasant 
emotion. The emotion stimulates the subcortex, perceptional sensitivity for the abnormal signal occurs in the subcortex. Then the vicious cycle is formed among the subcortex, the auditory cortex, and the cerebral limbic system. Moreover, the autonomic nervous system loses own auto-regulation function by excessive stimulation from the cerebral limbic system. The brain consequently recognizes the abnormal signal as infliction, i.e., the symptom of tinnitus arises.

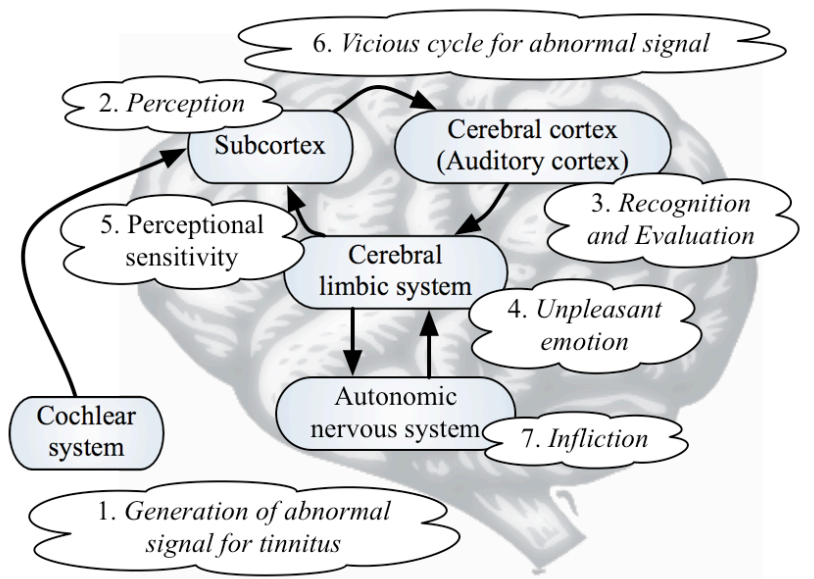

Fig. 1 The neurophysiological model for tinnitus generation

\section{Sound therapies for tinnitus}

Sufferers from tinnitus are treated by medication, sound therapy, therapy by electrical stimulation, mechanical aids, biofeedback, psychotherapy, and so on. As typical sound therapies, TM [4] and TRT [2, 3] were developed. Outcomes of the two therapies have been reported [5]. In TM, sound stimulation (masker) is applied to sufferers so that sound of tinnitus is masked. The type of stimulation is band noise. Its frequency band is determined by pitch matching test. Its amplitude is also determined by loudness balancing test. TRT consists of sound therapy and psychotherapy by directive counseling; its therapeutic purpose is to provoke habituation of brain for tinnitus. In the sound therapy, the type of stimulation is white noise, and its amplitude is smaller than the loudness of perceived tinnitus. Features of the two therapies are shown in Table 1.

\section{A neUral oscillator MODEL}

\section{A. Structure of the cerebral limbic system and its functions}

The cerebral limbic system is a nervous system in the brain. It consists of an amygdaloid complex, a hippocampus, and a hypothalamus. It also has connections to the hypothalamus, the frontal lobe, the temporal lobe, and the others in the brain [6]. Its architecture is illustrated in Fig. 2. The hippocampus manages memory formation and has a function to access to arbitrary memory. It connects to the hypothalamus via the medial forebrain bundle each other. The amygdaloid complex manages emotional formation by associating with the memories stored in the hippocampus. It also has bidirectional connection to the hypothalamus via the stria terminals. The hypothalamus controls the autonomic nervous system by production and secretory of hormone.

Table 1 Conditions of sound therapies and their clinical effects

\begin{tabular}{|c|c|c|}
\hline Item Therapy & TM & TRT \\
\hline \hline $\begin{array}{c}\text { Type of } \\
\text { sound stimulation }\end{array}$ & Band noise & White noise \\
\hline $\begin{array}{c}\text { Frequency of } \\
\text { sound stimulation }\end{array}$ & Same as tinnitus & - \\
\hline $\begin{array}{c}\text { Loudness of } \\
\text { sound stimulation }\end{array}$ & Same as tinnitus & $\begin{array}{c}\text { Smaller than } \\
\text { tinnitus }\end{array}$ \\
\hline Stimulated time & About 2 hours & About 7 hours \\
\hline $\begin{array}{c}\text { Appearance time } \\
\text { of clinical effect }\end{array}$ & Quickness & After some months \\
\hline $\begin{array}{c}\text { Sustained period } \\
\text { of clinical effect }\end{array}$ & Short & Long \\
\hline
\end{tabular}

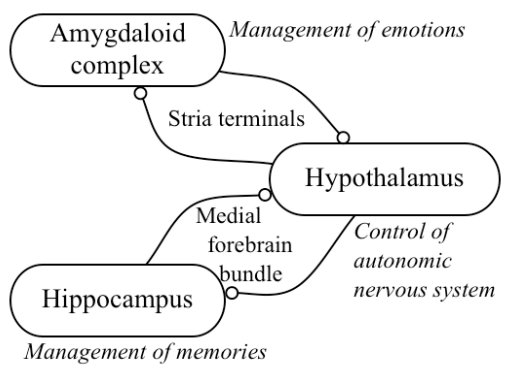

Fig. 2 Structure of cerebral limbic system

\section{B. An artificial neural model of the cerebral limbic system}

To account for clinical effect shown in Table 1 from the viewpoint of engineering, we discuss oscillation and its inhibition in an artificial cerebral model. We illustrate the architecture of the neural network model in Fig. 3. It was proposed as an approximation model for the amygdaloid kindling phenomenon [7]. The model consists of two excitatory neuronal units $E_{1}$ and $E_{2}$, and one inhibitory neuronal unit I. From the anatomical hypothesis, the $\mathrm{E}_{1}$ represents the excitatory aggregate of the amygdaloid complex. The $\mathrm{E}_{2}$ stands for the excitatory aggregate of the hypothalamus. The I represents the inhibitory aggregate of the hippocam- 
pus. The $E_{1}$ and $E_{2}$ have positive mutual coupling; its coupling coefficients are denoted by $C_{21}$ and $C_{12}$. The $\mathrm{E}_{2}$ and the I also have mutual coupling with the coefficients, $C_{I 2}$ and $C_{2 I}$. Note that the values of the $C_{I 2}$ and $C_{2 I}$ are positive and negative, respectively. The $S$ represents external stimulation to the $\mathrm{E}_{1}$. Its dynamics is described by

$$
\begin{aligned}
& \frac{d x_{1}}{d t}=\left(-x_{1}+C_{12} z_{2}+S\right) / \tau_{1} \\
& \frac{d x_{2}}{d t}=\left(-x_{2}+C_{21} z_{1}-C_{2 I} z_{I}\right) / \tau_{2} \\
& \frac{d x_{I}}{d t}=\left(-x_{I}+C_{I 2} z_{2}\right) / \tau_{I} \\
& z_{j}=\frac{2}{\pi} \tan ^{-1} x_{j},
\end{aligned}
$$

where $x_{j}, z_{j}$, and $\tau_{j}(j=1,2, I)$ are the state, the output, and the time constant in each unit, respectively. The $C_{j k}(k=1,2$, $I$ ) denotes the coupling coefficient from the $k$-unit to the $j$ unit. Although actual synapses in a brain intrinsically have plasticity and feature adjustability of the brain, many works have been done on the analysis of coupled neural oscillator models with constant coupling [7, 8]. As mentioned above, TRT is to retrain the brain of sufferers from tinnitus based on the plastic modification of synaptic connections. Therefore, this paper employs the neural model with a plastic coupling defined by

$$
\frac{d C_{12}}{d t}=\left(-C_{12}+b z_{1} z_{2}+C_{0}\right) / \tau_{c}
$$

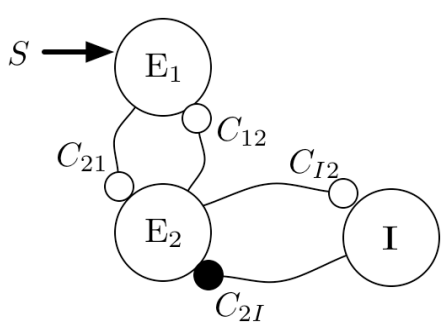

Fig. 3 A neural model of the cerebral limbic system

\section{OSCILLATION AND ITS INHIBITION}

\section{A. Appearance of oscillation without external stimulation}

In this paper we fixed the parameters such that $\tau_{1}=10$, $\tau_{2}=10, \tau_{I}=20, \tau_{c}=500, C_{21}=10, C_{I 2}=20, C_{2 I}=10, b=20$, and $C_{0}=5$. Then we consider the situation without external stimulation, i.e., $S=0$ for all $t$. We observed a stationary oscillation in that the initial state is $\left(x_{1}, x_{2}, x_{I}, C_{12}\right)=(0.1,0.0$,
$0.0,11.8)$. The waveforms of the oscillation are shown in Fig. 4. We also observed a stationary equilibrium (nonoscillatory state) under the same parameters; but the initial state is $\left(x_{1}, x_{2}, x_{I}, C_{12}\right)=(0.0,0.0,0.0,11.8)$. This equilibrium is at the origin, which is illustrated in Fig. 5. Thus the stable oscillation and the stable equilibrium coexist under the same parameters, hence, the neural model has the bi-stable state. We assume that the oscillatory state and the non-oscillatory state are associated with appearance and disappearance of tinnitus, respectively. Then the bi-stable state could explain the fact that appearance and disappearance of tinnitus. Note that we can investigate the region of parameters that the bistable state exists by computing bifurcation sets of the oscillation and the equilibrium. The investigation will be conducted.

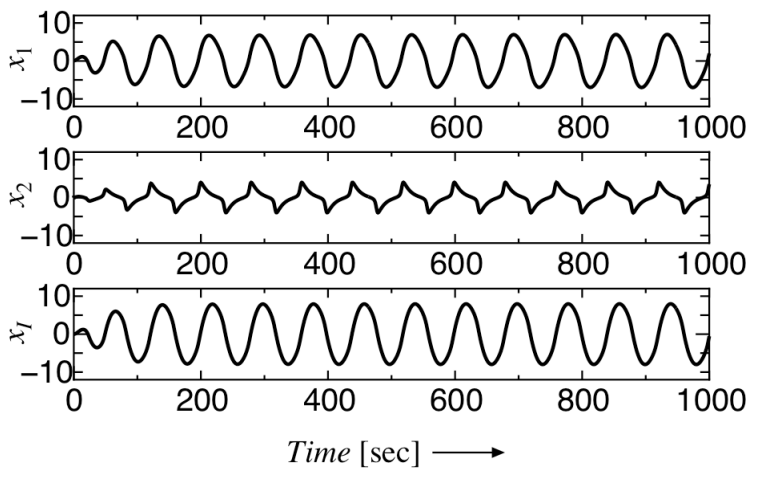

Fig. 4 Oscillatory state

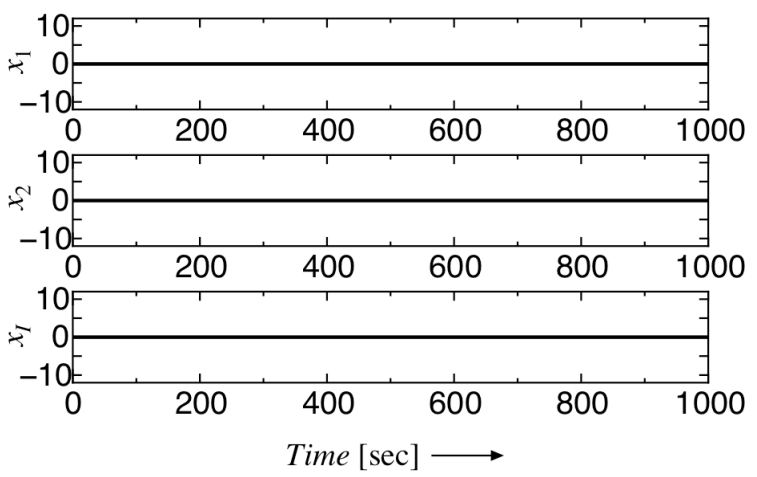

Fig. 5 Non-oscillatory state

\section{B. Inhibition of oscillation by external stimulation}

Let us demonstrate that the oscillation can be inhibited by external stimulation which is sinusoidal stimulation defined by

$$
S=V_{e} \sin 2 \pi f_{e} t .
$$


We show numerical simulation results for two sinusoidal stimuli with different frequency in Figs. 6-7. Figure 6 shows a successful example of inhibition of the oscillation. We fixed the parameter of the stimulation as $V_{e}=2$ and $f_{e}=0.01$; we provided the stimulation in only the time from 500 to $2500 \mathrm{sec}$. Note that the value of the plastic coupling $C_{12}$ slowly decreases by the stimulation, that is, the neural model has been retrained so that the oscillation disappears. Figure 7 shows an unsuccessful example of inhibition of the oscillation. Only the frequency of the stimulation is changed as $f_{e}=0.015$. Throughout the simulation, the oscillation always appears, and the $C_{12}$ keeps high value. Hence, the retraining effect of the stimulation was not observed.

\section{CONCLUding REMARKS}

To account for the mechanism of perceiving tinnitus and the effect of sound therapy from the viewpoint of engineering, we proposed a neural oscillator model with a plastic coupling for the cerebral limbic system. We observed that an oscillatory state and a non-oscillatory state coexist in a certain parameter set. It is also discovered that the oscillation can be inhibited by external sinusoidal stimulation. The phenomena could explain the mechanism of tinnitus generation and the fact that the retrained cerebral limbic system temporally stops perceiving tinnitus after sound therapy.

The coexisting region of the oscillation and the equilibrium by computing their bifurcation sets will be investigated. We will also study the neural oscillator model with which stimulation of white noise or band noise is provided.

\section{REFERENCES}

1. Axelsson A. and Ringdahl A. (1989) Tinnitus-a study of its prevalence and characteristics. Br J Audiol 23(1): 53-62

2. Jastreboff P. J. (1990) Phantom auditory perception (tinnitus): mechanisms of generation and perception. Neurosci Res. 8(4): 221254

3. Jastreboff P. J. and Jastreboff M. M. (2000) Tinnitus Retraining Therapy (TRT) as a method for treatment of tinnitus and hyperacusis patients. J Am Acad Audiol. 11(3):162-177

4. Hazell J. W. and Wood S. (1981) Tinnitus masking-a significant contribution to tinnitus management. Br J Audiol. 15(4):223-230

5. Henry J. A., Schechter M. A. et al. (2006) Outcomes of clinical trial: tinnitus masking versus tinnitus retraining therapy. J Am Acad Audiol. 17(2):104-132

6. Limbic system at http://www.healing-arts.org/n-r-limbic.htm

7. I. Lieblich and S. Amari (1978) An Extended First Approximation Model for the Amygdaloid Kindling Phenomenon, Biol. Cybernetcs, 28:129-135

8. Michael A.A. (1995) The Handbook of Brain Theory and Neural Networks. The MIT Press, London
Address of the corresponding author:

Author: Dr. Ken'ichi FUJIMOTO

Institute: Faculty of Medicine, The University of Tokushima

Street: $\quad 3-18-15$ Kuramoto-cho

City: Tokushima

Country: Japan

Email: fujimoto@medsci.tokushima-u.ac.jp
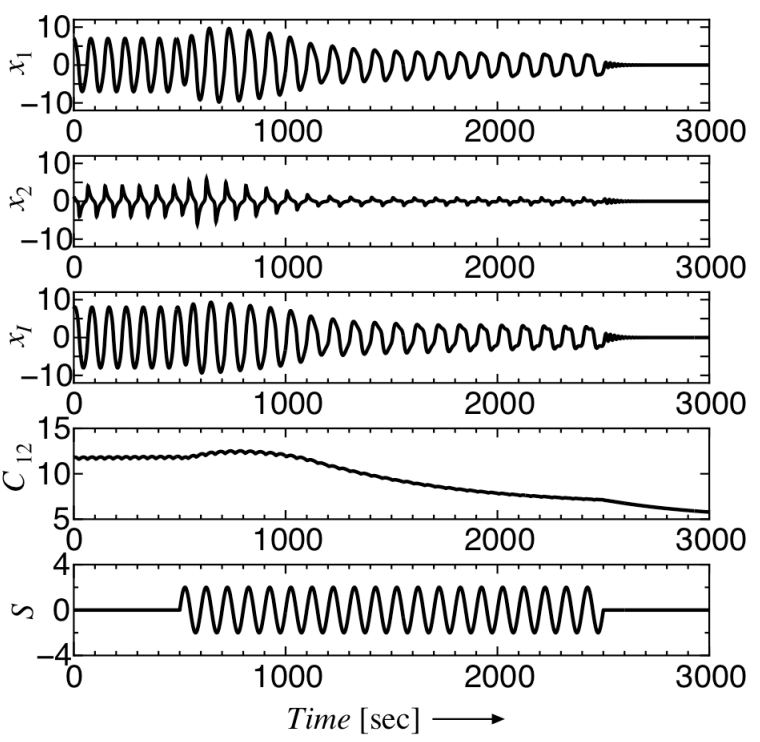

Fig. 6 A successful example of inhibition of the oscillations
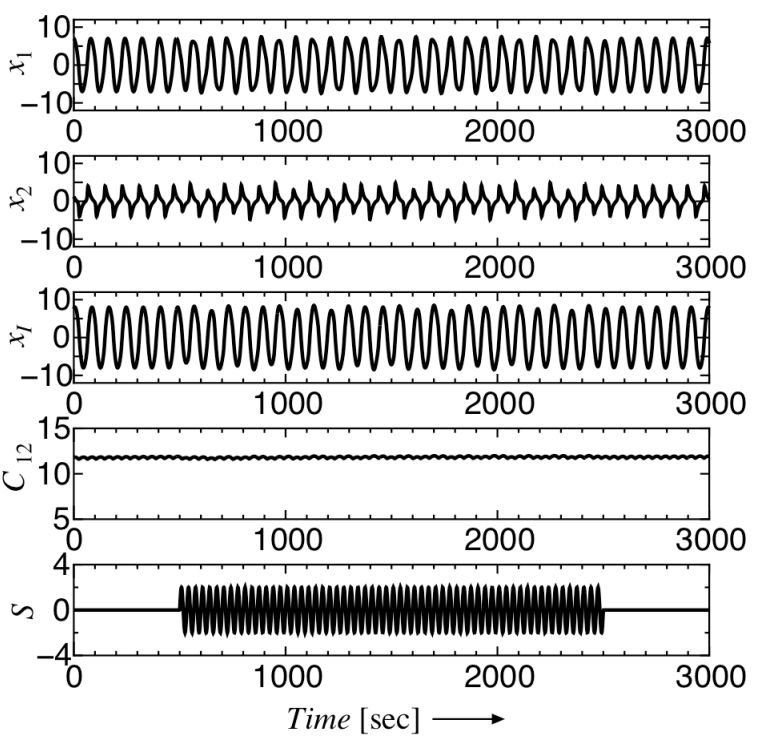

Fig. 7 An unsuccessful example of inhibition of the oscillation 